Revista de
Economild
Contemporâned

\title{
MÚLTIPLO EMPREGO NO BRASIL: UMA ANÁLISE DA OFERTA DE TRABALHO USANDO DADOS DA PNAD
}

\author{
Daniel Tomaz de Sousa ${ }^{a}$ \\ Francisco Germano Carvalho Lúcio ${ }^{b}$ \\ Leandro de Almeida Rocco ${ }^{c}$
}

\begin{abstract}
${ }^{a}$ Estudante de doutorado do Programa de Pós-Graduação em Economia Aplicada da Universidade Federal da Paraíba (PPGE/UFPB). João Pessoa, PB, Brasil. ORCID: https://orcid.org/0000-0003-2530-2593.

bEstudante de doutorado do Programa de Pós-Graduação em Economia da Universidade Federal do Ceará (CAEN/UFC). Fortaleza, CE, Brasil. ORCID: https://orcid.org/0000-0001-9153-4849.

'Professor do Programa de Pós-Graduação em Economia da Universidade Federal do Ceará (CAEN/ UFC). Fortaleza, CE, Brasil. ORCID: https://orcid.org/0000-0002-9325-3239.
\end{abstract}

Artigo recebido em 26/02/2018 e aceito para publicação em 06/12/2018.

RESUMO: O presente estudo tem como objetivo analisar os determinantes da oferta de trabalho no mercado do múltiplo emprego no Brasil. Foram utilizados dados da Pesquisa Nacional por Amostra de Domicílios (PNAD), dos anos de 2004 e 2014, e um modelo com variável dependente limitada, tobit. Os resultados indicam que o mercado em análise é composto em sua maioria por homens, chefes de família e trabalhadores que têm um nível educacional mais elevado. Da função oferta estimada tem-se que indivíduos do sexo masculino ofertam mais horas de trabalho. Dos aspectos teóricos testados, o motivo restrição de horas foi relevante e reduz a oferta. Já o motivo insegurança atua aumentando a oferta de trabalho.

PALAVRAS-CHAVE: múltiplo emprego; oferta de trabalho; modelo tobit.

CLASSIFICAÇÃO JEL: J21; J22. 


\title{
MULTIPLE JOB HOLDING IN BRAZIL: AN ANALYSIS OF LABOR SUPPLY USING PNAD DATA
}

\begin{abstract}
The present study has as objective to analyze the determinants of labor supply in the multiple job market in Brazil. We use data from the National Household Sample Survey (PNAD), from 2004 and 2014, and a model with a limited dependent variable, tobit. The results indicate that the market in question is composed mostly of men, heads of families and workers who have a higher educational level. From the estimated supply function, it is shown that male individuals offer more hours of work. From the theoretical viewpoints tested, the reason for restricting hours was relevant and reduced the supply and the reason for age insecurity increases a job offer.
\end{abstract}

KEYWORDS: multiple job; labor supply; tobit model. 


\section{INTRODUÇÃO}

Na perspectiva da oferta de trabalho, tem-se observado que há uma parcela da força de trabalho que está ofertando horas não apenas na ocupação principal, mas também em uma segunda ocupação. Diz-se que o trabalhador ocupado nesse segmento do mercado pertence ao mercado de trabalho secundário. ${ }^{1}$ Segundo Casari e Bacha (2011), nos anos de 2004 a 2009, em média 4,41\% dos trabalhadores tinham dois empregos no Brasil.

Há diversas abordagens para explicar as razões que determinam a escolha de ter uma ocupação adicional. Destacam-se dentre os enfoques teóricos a restrição de horas no trabalho principal de Shishko e Rostker (1976), o modelo de trabalho heterogêneo, proposto por Conway e Kimmel (1998) e o modelo de insegurança no trabalho principal, desenvolvido por Bell, Hart e Wright (1997).

Para Shishko e Rostker (1976), o desejo de trabalhar mais horas pode levar a pessoa a buscar uma fonte alternativa de trabalho, quando essas horas adicionais não estão disponíveis no emprego principal.

$\mathrm{Na}$ abordagem descrita por Bell, Hart e Wright (1997), o segundo emprego funciona como um meio de dirimir os riscos associados ao emprego principal. Por outro lado, ter um contrato de trabalho mais seguro diminui a busca por outras ocupações, como destacado por Böheim e Taylor (2004). Entretanto, Wu, Braimbridge e Zu (2009) não encontram evidências de que a insegurança no trabalho principal tenha impacto na decisão de participar no mercado do segundo emprego.

O modelo de trabalho heterogêneo, desenvolvido por Conway e Kimmel (1998), é centrado na ideia de benefícios não pecuniários associados ao emprego adicional, em que se busca um maior nível de utilidade. Outras causas ${ }^{2}$ podem ser enumeradas para justificar a participação no mercado secundário.

Como exemplos de trabalhos empíricos destacam-se Averett (2001), Foley (1997), Heineck e Schwarze (2004), Böheim e Taylor (2004), Guariglia e Kim (2006), Panos, Pouliakas e Zangelidis (2014) e Atherton et al. (2016).

No Brasil, Menezes e Carrera-Fernandez (2003) estudam o comportamento do segundo emprego na região metropolitana de Salvador, sem se atentar a modelos teóricos. Já Casari e Bacha (2011) utilizam o modelo teórico e aplicam a dados da Pesquisa Nacional por Amostra de Domicílios (PNAD) e estimam a oferta de horas para a segunda ocupação, entretanto não fazem uso do desenho amostral complexo da pesquisa.

\footnotetext{
1 Não há uma terminologia padrão para estudos brasileiros, já que há escassez de pesquisa sobre esse mercado, assim, porventura, como sinônimo do mercado secundário serão usados os seguintes termos: mercado do múltiplo emprego e/ou mercado do segundo emprego para designar situações semelhantes e quando se fizer necessário, maiores detalhes serão expostos para que fique claro ao leitor.

2 Ver Atherton et al. (2016) e Heineck e Schwarze (2004).
} 
Apesar da existência de um contingente expressivo de trabalhadores no mercado secundário, e de uma literatura consolidada, pouco se sabe dos motivos que levam os indivíduos a realizar essa prática no Brasil. O conhecimento da composição da força de trabalho de um país é um importante subsídio para o desenho de políticas. Além de que a regulamentação do trabalho tem impacto nas decisões dos agentes, como destacado por Renna (2006).

A partir do exposto, este estudo visa identificar quais fatores contribuem para que os trabalhadores ofertem trabalho no mercado das segundas ocupações no Brasil. Além disso, objetiva traçar o perfil do moonlighter. ${ }^{3}$

Além desta introdução, o estudo divide-se em mais três seções. A seção dois apresenta uma breve revisão de literatura. Seção três aborda questões metodológicas. A seção quatro trata dos resultados e discussão. As considerações finais encontram-se na seção cinco.

\section{MÚLTIPLO EMPREGO: TEORIA E EVIDÊNCIAS EMPÍRICAS}

\subsection{DETERMINANTES DO MÚLTIPLO EMPREGO}

Há diversas abordagens para explicar as razões que são determinantes na escolha de ter uma ocupação adicional. O trabalho seminal de Shishko e Rostker (1976) modela a restrição de horas do trabalho principal, o modelo está alinhado na ideia de que o trabalhador deseja trabalhar mais horas, e essas horas adicionais não estão disponíveis no emprego principal. Dessa forma, o único meio para trabalhar mais é um segundo emprego. Segundo Kimmel e Conway (2001), essa restrição limita os ganhos possíveis no trabalho principal e provoca dificuldade financeira.

Já o modelo de trabalho heterogêneo de Conway e Kimmel (1998) não exclui o motivo descrito anteriormente, e sim adiciona mais robustez a análise. Baseia-se na ideia de que benefícios não pecuniários podem influenciar na decisão de participação no mercado do segundo emprego. Uma das hipóteses mais fortes levantadas pelos autores é a de que trabalhadores que executam trabalhos principais com pouca variabilidade de funções são mais propensos a buscar uma segunda ocupação, e fazem isso em busca de um maior nível de utilidade.

\footnotetext{
3 Termo utilizado na literatura internacional para o trabalhador alocado no mercado do múltiplo emprego.
} 
O modelo descrito por Bell, Hart e Wright (1997) insere a insegurança no trabalho principal como propulsor da ida ao mercado secundário. Nessa abordagem, o segundo emprego funciona como um meio de dirimir os riscos associados ao emprego principal. Por outro lado, ter um contrato de trabalho mais seguro diminui a busca por outras ocupações, como destacado por Böheim e Taylor (2004).

Além dos modelos discutidos anteriormente, outras causas podem ser enumeradas para justificar a participação no mercado secundário. Atherton et al. (2016) se baseiam na visão de que as pressões financeiras são um incentivo para o segundo emprego, além de testarem a relevância dos custos domésticos.

Heineck e Schwarze (2004) evidenciam que os trabalhadores também podem estar buscando segundas ocupações à procura de aquisição de novas habilidades e ganhos de experiência que, de certa forma, não eram possíveis de serem adquiridas na ocupação principal.

\subsection{EVIDÊNCIAS EMPÍRICAS}

Kimmel e Conway (2001) verificam que o moonlighter dos EUA continua um pouco mais pobre do que o trabalhador com apenas um emprego, apesar de trabalhar tempo integral no trabalho primário e parte em um segundo emprego que paga menos que o principal. $\mathrm{O}$ fato de o trabalhador ainda ser mais pobre evidencia que ter um segundo emprego não necessariamente elimina as dificuldades financeiras do indivíduo.

Averett (2001) examina as razões que levam o indivíduo a ter um segundo emprego com foco na diferença de gênero. As razões de entrada no mercado secundário para ambos os sexos são similares, renda do não trabalho tem efeito negativo, tanto para homens quanto para as mulheres, a idade tem um efeito positivo apenas para homens. Outro resultado interessante encontrado por Averett (2001) foi a existência de uma correlação negativa entre a entrada na força de trabalho e a decisão de participar do mercado secundário.

Foley (1997) e Guariglia e Kim (2006) estudam o fenômeno do múltiplo emprego na Rússia. $\mathrm{O}$ primeiro verificou que os mais propensos a possuírem segundas ocupações são homens, residentes urbanos e indivíduos com nível de educação mais elevado. Já Guariglia e Kim (2006) verificaram a presença de transitoriedade - as pessoas passam pouco tempo nesse segundo emprego -, e, em alguns casos, há chance de o segundo emprego tornar-se o principal, situação já proposta por Shishko e Rostker (1976), que ainda encontram que níveis elevados de educação reduzem a participação nesse mercado.

Heineck e Schwarze (2004) e Heineck (2009) usam dados do Reino Unido e Alemanha para comparar o comportamento do múltiplo emprego intergênero entre os países. Há evidências de similaridades e discrepâncias entre os países. Os efeitos do salário prin- 
cipal e da renda do não trabalho são mais fortes para o caso do Reino Unido, já o efeito da restrição de horas é encontrado apenas para homens da Alemanha.

Para dados do Reino Unido dos anos 1990 há uma persistência do múltiplo emprego ao longo do tempo, além disso, os trabalhadores permanecem cerca de dois anos nessa segunda ocupação. A oferta é sensível a maiores níveis de capital humano, que agem de forma positiva na oferta; alguns dos fatores que reduzem a oferta de horas de trabalho são contratos mais seguros (um dos meios de qualificar o motivo insegurança do trabalho principal) e a restrição de horas no trabalho principal (BÖHEIM e TAYLOR, 2004).

No Reino Unido, ser do setor público encoraja participar do mercado secundário, e, tanto para homens quanto para mulheres, a idade tem um impacto negativo. Para as mulheres, ser casada diminui a oferta de horas (WU, BRAIMBRIDGE e ZU, 2009).

Analisando a dinâmica do duplo emprego no Reino Unido, Panos, Pouliakas e Zangelidis (2014) tentam relacionar escolha e mobilidade ocupacional com a decisão de participar do mercado secundário. Encontram evidências de que os indivíduos podem estar usando o multiemprego como um canal de obtenção de novas habilidades e competências e também como condutor para novas carreiras, pois os moonlighters são duas vezes mais propensos a mudar para o autoemprego do que aqueles que têm apenas uma ocupação.

Martinez Jr. et al. (2014) estudam o comportamento do múltiplo emprego na Indonésia por meio de um modelo logit. Dentre alguns dos resultados encontrados, tem-se que homens são mais propensos a ter um emprego adicional, assim como indivíduos casados e chefes de família. Dos fatores que reduzem as chances de participação destacam-se: ser residente urbano, número de horas trabalhadas e rendimento do emprego principal. Idade tem um efeito positivo, contudo a taxas são decrescentes.

Atherton et al. (2016), ao contrário de Böheim e Taylor (2004), não excluem os trabalhadores independentes da amostra e fazem análise comparando-os com os empregados. Como os modelos teóricos não dão suporte a análises de trabalhadores independentes, os autores estendem o modelo para o caso dos trabalhadores por conta própria. Além disso, acrescentam variáveis que indicam dificuldade financeira e custos de habitação.

Dentre os resultados encontrados por Atherton et al. (2016) destaca-se que as mulheres são mais propensas ao autoemprego, contudo, para algumas, é necessário um segundo emprego para complementar a renda. Homens com ocupação principal autônoma trabalham mais horas no segundo emprego, e a dificuldade financeira é um dos condutores da entrada no mercado do segundo emprego tanto para homens independentes quanto empregados e também para mulheres independentes.

$\mathrm{Na}$ literatura nacional existem poucos estudos nessa área. Menezes e Carrera-Fernandez (2003) e Casari e Bacha (2011) são os autores que pesquisaram sobre a temática da participação e oferta no mercado secundário. 
Menezes e Carrera-Fernandez (2003) estudaram a formação do rendimento da segunda ocupação e analisaram os determinantes da participação dos trabalhadores no mercado do segundo emprego na região metropolitana de Salvador. Tem-se, pela pesquisa, que os mais propensos ao segundo emprego são mulheres, chefes de família e os assalariados, e que buscam esse emprego adicional para complementar a renda devido aos baixos ganhos do trabalho principal.

Casari e Bacha (2011) estudam em nível nacional o comportamento do mercado secundário e estimam a oferta de horas para a segunda ocupação. Encontram que o fato de as ocupações serem heterogêneas amplia a oferta, revelando existência de benefícios não pecuniários do trabalho. Estabilidade no trabalho principal e ser servidor público têm um efeito positivo na oferta. Ser residente de zona urbana e de regiões metropolitanas têm impacto negativo sobre a oferta.

Pelas evidências descritas percebe-se a relevância das pessoas que optam por ofertar horas de trabalho no mercado secundário, assim, este estudo tenta diminuir as lacunas existentes na literatura nacional acerca desse tema de pesquisa. Além da contribuição empírica, pretende-se ampliar os estudos na área.

\subsection{MODELO TEÓRICO}

Esta seção tem como objetivo, com base na literatura, expor uma versão simplificada do modelo teórico sobre múltiplo emprego. Tomam-se como base Conway e Kimmel (1998) e Böheim e Taylor (2004).

Assume-se que as decisões do agente em relação à escolha do trabalho secundário derivam do processo de maximização de utilidade. A função utilidade pode ser escrita como:

$$
U\left(C, h_{1}, h_{2}, L, Z\right)
$$

Em que $C$ denota consumo; e $h_{1}, h_{2}$ indicam o número de horas trabalhadas no emprego principal e secundário, respectivamente; $L$ denota lazer e $Z$ é um vetor de variáveis socioeconômicas que podem afetar o nível de utilidade.

A função utilidade descrita por (1) é maximizada, sujeita a restrição orçamentária e a uma restrição temporal:

$$
\begin{gathered}
C=w_{1} h_{1}+w_{2} h_{2}+Y \\
T=h_{1}+h_{2}+L
\end{gathered}
$$

Os salários da ocupação principal (secundária) são denotados por $w_{1}\left(w_{2}\right)$. $Y$ é a renda do não trabalho e $T$ é o total de tempo disponível. Em adição há as usuais restrições de não negatividade em $h_{i}, L, C$. 
Substituindo as restrições (2) e (3) em (1) para $C$ e $L$, o problema de maximização torna-se:

$$
\max _{h 1, h 2} U\left(w_{1} h_{1}+w_{2} h_{2}+Y, h_{1}, h_{2}, T-h_{1}-h_{2}, Z\right)
$$

Caso o trabalhador deseje ofertar mais horas do que é possível no trabalho principal, $h_{1}$ não é mais uma variável de escolha. Dessa forma, a decisão de ter um segundo emprego, dado um número fixo de horas do primeiro emprego, $\left(\underline{h_{1}}\right)$ dependerá da utilidade marginal do segundo emprego.

Fazendo $h_{1}=\underline{h_{1}}$ no problema de maximização definido por (4), e resolvendo, obtém-se a seguinte relação no ótimo:

$$
\frac{U_{2}-U_{L}}{U_{C}}=-w_{2}
$$

Em (5), $U_{2}, U_{L}$ e $U_{C}$ representam as derivadas parciais em relação a $h_{2}, L$ e $C$, respectivamente. $\mathrm{O}$ numerador de (5) é a desutilidade marginal de uma hora a mais no segundo emprego. Resolvendo de forma implícita, encontra-se a seguinte equação de horas ofertadas para o segundo emprego para aqueles que têm restrições de horas no emprego principal:

$$
h_{2}=h_{2}^{R}\left(Z, \underline{h_{1}}, w_{2}, Y\right)
$$

Em que $h_{2}^{R}$ é utilizado para indicar que os trabalhadores sofrem de restrição de horas. Da equação (6) tem-se que $\frac{\partial h_{2}^{R}}{\partial Y}<0$, pois o lazer é assumido como um bem normal; $\frac{\partial h_{2}^{R}}{\partial \overline{h_{1}}}<0$, que nos diz que, quanto maior o tempo despendido no trabalho principal, menor a oferta de horas do segundo emprego, e $\frac{\partial h_{2}^{R}}{\partial w_{2}}$ tem sinal ambíguo por
causa dos efeitos renda e substituição.

Se o indivíduo decide ter uma segunda ocupação porque os trabalhos são heterogêneos (tem diferentes benefícios ou custos não pecuniários) e não porque sofre restrição de horas, como destacado anteriormente, ambas as horas de trabalho são variáveis de escolha, tanto as da ocupação principal $\left(h_{1}\right)$ quanto as da secundária $\left(h_{2}\right)$. Agora, do problema maximização, obtêm-se as seguintes condições de ótimo:

$$
\frac{U_{i}-U_{L}}{U_{C}}=-w_{i}, \quad i=1,2
$$

A equação (7) implica que as horas são fornecidas para qualquer trabalho até que a desutilidade do trabalho seja igual ao negativo do salário $i$. Resolvendo para $h_{1}$ e $h_{2}$ : 


$$
\begin{aligned}
& h_{1}=h_{1}^{N R}\left(Z, w_{1}, w_{2}, Y\right) \\
& h_{2}=h_{2}^{N R}\left(Z, w_{1}, w_{2}, Y\right)
\end{aligned}
$$

Em que $h_{i}^{N R}$ denota que o trabalhador não enfrenta restrição de horas. A estática comparativa do modelo sugere que $\frac{\partial h_{i}^{N R}}{\partial w_{j}}<0$ para $i \neq j$, por exemplo, quando $i=1$ e $j=2$, implica que um maior salário no segundo emprego faz com que a oferta de horas no primeiro emprego diminua, com sinal ambíguo quando $i=j$. Novamente, como o lazer é um bem normal, $\frac{\partial h_{i}^{N R}}{\partial Y}<0$.

Neste artigo a análise é limitada a indivíduos com restrição de horas, contudo, mesmo nessa perspectiva, motivos ligados a ganhos marginais de utilidade podem estar presentes na escolha dos agentes. Como destacado por Heineck (2009), ambos os motivos podem estar influenciando na decisão dos agentes, e não apenas um deles, exclusivamente. Na seção que segue a estratégia empírica e os dados são apresentados.

\section{METODOLOGIA}

\subsection{ESTRATÉGIA ECONOMÉTRICA}

Há duas formas de tratar os trabalhadores alocados no mercado secundário, como destacado no modelo teórico. Ou o indivíduo sofre de restrição de horas, e/ou busca diversificação nos trabalhos (motivo heterogeneidade). Este trabalho segue Böheim e Taylor (2004), no sentido de que é realizada uma análise para os indivíduos que sofrem de restrição de horas na ocupação principal, sendo essas horas $\left(h_{1}\right)$ uma variável dada e não mais uma variável de escolha. Estima-se uma função oferta com o seguinte formato:

$$
h_{2}=h_{2}(X)
$$

Em (10), $X$ é vetor de variáveis independentes.

A variável $h_{2}$ está disponível apenas para os indivíduos alocados no mercado de trabalho secundário. Contudo, as covariáveis estão disponíveis para toda a amostra. Como temos uma equação com variável dependente limitada, o método adequado a esse caso é o tobit, descrito a seguir:

$$
y_{i}^{*}=x_{i} \beta+\varepsilon_{i}
$$

Em que $y_{i}^{*}$ é uma variável latente, que é parcialmente observada. Note que $y_{i}$ é observada e definida da seguinte forma: 


$$
y_{i}=\left\{y_{i}^{*} \text { se } y_{i}^{*}>00, \text { se } y_{i}^{*}=0\right.
$$

O efeito marginal ${ }^{4}$ do tobit pode ser expresso da seguinte forma:

$$
\frac{\partial E\left[\frac{y_{i}}{x_{i}}\right]}{\partial x_{i}}=\beta \Phi\left(\frac{x_{i}^{\prime} \beta}{\sigma}\right)
$$

Em (13), $\Phi($.$) é a função de distribuição da normal padronizada. Note, pela equa-$ ção (13), que variações em $x_{i}$ têm efeito não somente sobre a média da variável observada, mas também sobre a probabilidade da variável ser observada.

\subsection{DADOS}

Os dados utilizados nesta pesquisa são provenientes da Pesquisa Nacional por Amostra de Domicílios (PNAD) realizada anualmente, exceto em anos censitários, pelo Instituto Brasileiro de Geografia e Estatística (IBGE). A escolha se deve ao fato de que essa pesquisa contém informações que podem classificar os indivíduos de acordo com suas características individuais, de localização e de trabalho. Foram selecionadas as PNAD de 2004 e 2014 para verificar se há um comportamento diferente ao longo do tempo.

O grupo de interesse da pesquisa é composto por pessoas que possuem, além da principal, uma ocupação adicional, sendo os rendimentos dessas ocupações auferidos em dinheiro.

A Tabela 1 mostra a frequência relativa dos indivíduos com uma, duas ou mais de duas ocupações nos respectivos anos analisados. Em 2004, pessoas com duas ocupações representam 4,38\% da força de trabalho, e em 2014 esse valor é de 3,25\%; quantidades consideradas expressivas que reforçam a relevância do estudo. É possível identificar pessoas que têm mais de duas ocupações. Contudo não há como classificar essas ocupações e nem saber o quanto de remuneração fornecem, logo, foram desconsideradas.

O Quadro 1, no Apêndice A, descreve as variáveis utilizadas no estudo, que foram selecionadas tendo como base a literatura sobre o múltiplo emprego.

Foram considerados indivíduos com idade entre 15 e 65 anos. Em relação à posição na ocupação no trabalho principal, foram considerados apenas empregados e com remuneração. Por não satisfazerem à condição de restrição de horas no trabalho principal,

\footnotetext{
4 O efeito marginal para a variável latente é o próprio coeficiente estimado do modelo tobit.
} 
trabalhadores domésticos, ${ }^{5}$ empregadores e trabalhadores por conta própria foram excluídos da amostra. Nota-se que, nesses empregos ou na situação de empregador, a liberdade de alocação de horas é maior, logo, tratá-los como os demais empregados tornaria a estimação enviesada. Destaca-se ainda que os indivíduos podem exercer as ocupações citadas em seu segundo emprego; a exclusão é realizada apenas na ocupação principal.

Tabela 1 - Número de trabalhos na semana, Brasil, 2004/2014

\begin{tabular}{lcccc}
\hline & \multicolumn{2}{c}{$\mathbf{2 0 0 4}$} & \multicolumn{2}{c}{$\mathbf{2 0 1 4}$} \\
\hline Número de Trabalhos & Frequência & Percentual & Frequência & Percentual \\
\hline Uma ocupação & 77.222 .781 & 95,25 & 95.948 .748 & 96,48 \\
Duas ocupações & 3.553 .477 & 4,38 & 3.234 .936 & 3,25 \\
Três ou mais ocupações & 300.091 & 0,37 & 263.318 & 0,26 \\
\hline
\end{tabular}

Nota: Amostra expandida.

Fonte: Elaboração própria com base em dados da pesquisa.

Uma alternativa para trabalhar com indivíduos que não têm limitação de horas na ocupação caracterizada como principal é fazer uma análise em separado, assim como feito por Atherton et al. (2016), que fazem comparação destes com empregados.

\subsection{PLANO AMOSTRAL COMPLEXO ${ }^{6}$}

A PNAD é uma pesquisa realizada por amostragem complexa, pois envolve estratificação, conglomeração com um, dois ou três estágios de seleção, dependendo do estrato, como destacado por Silva, Pessoa e Lila (2002).

Santos (2009) destaca que a análise descritiva de dados da PNAD sofre influência dos pesos amostrais, contudo, quando o interesse é a estimação da variância, esta sofre tanto influência dos pesos amostrais quanto, conjuntamente, da estratificação e conglomeração. Dessa forma, é indispensável levar em consideração o desenho amostral complexo para obter estimativas não viciadas das variâncias.

Usam-se, então, as seguintes variáveis para caracterizar o plano amostral complexo: STRAT (estrato), PSU (Unidade Primária de Amostragem) e Peso (V4729), disponíveis em ambos os anos analisados, 2004 e 2014. Para estimar a variância e os desvios-padrão, optou-se pela linearização de Taylor.

5 Salienta-se que, nos períodos estudados no artigo, não havia a legislação mais recente sobre o trabalho doméstico, que pode alterar a oferta de trabalho.

6 Para mais detalhes sobre o plano amostral complexo da PNAD, ver Santos (2009), Carvalho, Neri e Silva (2006) e Silva, Pessoa e Lila (1998). 
A estimação do modelo tobit, devido ao uso do plano amostral complexo, é realizada por meio do método da Máxima Pseudoverossimilhança (MPV).

Os valores do DEFF (design effect) e MEFF (mis specification effect) que avaliam o impacto do desenho amostral (efeito do plano amostral - EPA) na estimação do modelo são também calculados e utilizados para reforçar a relevância do desenho amostral da PNAD. Valores maiores que um (do MEFF ou DEFF) indicam que ignorar o plano amostral leva a subestimar a variância do estimador; valores menores que um indicam superestimação ao desconsiderar o desenho amostral; valores iguais a um revelam que não há diferenças entre as variâncias estimadas (SILVA, PESSOA e LILA, 2002).

\section{RESULTADOS E DISCUSSÃO}

\subsection{PERFIL DO MOONLIGHTER}

Nesta seção serão expostas características para determinar o perfil do moonlighter no Brasil. A Tabela 2 mostra que, em ambos os anos, os homens e os indivíduos de cor branca representam a maioria dos moonlighters. Há uma redução no número de componentes da família. Como destacado por Leone, Maia e Baltar (2010), essa é uma característica que a população vem adquirindo ao longo do tempo.

Tabela 2 - Perfil demográfico do moonlighter, Brasil, 2004/2014 (*)

\begin{tabular}{|c|c|c|c|c|}
\hline \multirow{2}{*}{ Característica } & \multicolumn{2}{|c|}{2004} & \multicolumn{2}{|c|}{2014} \\
\hline & Média & DP & Média & DP \\
\hline Sexo & 0,60 & 0,49 & 0,55 & 0,50 \\
\hline Raça & 0,53 & 0,50 & 0,50 & 0,50 \\
\hline Componentes & 3,71 & 1,58 & 3,19 & 1,30 \\
\hline Chefe & 0,63 & 0,48 & 0,61 & 0,49 \\
\hline Centro-Oeste & 0,06 & 0,23 & 0,07 & 0,25 \\
\hline Nordeste & 0,38 & 0,48 & 0,29 & 0,46 \\
\hline Norte & 0,10 & 0,29 & 0,06 & 0,24 \\
\hline Sudeste & 0,30 & 0,46 & 0,39 & 0,49 \\
\hline Sul & 0,17 & 0,37 & 0,18 & 0,39 \\
\hline RM & 0,20 & 0,40 & 0,23 & 0,42 \\
\hline Urbano & 0,73 & 0,44 & 0,83 & 0,37 \\
\hline Migrante & 0,36 & 0,48 & 0,53 & 0,50 \\
\hline Idade & 38,34 & 11,09 & 40,50 & 11,50 \\
\hline Estudo (anos) & 8,26 & 5,24 & 11,47 & 4,52 \\
\hline
\end{tabular}

Notas: (*) A diferença entre as variáveis foi avaliada pela estatística t. (a) Média ou Proporção; (b) DP= desvio-padrão; (c) Amostra expandida; (d) A variável chefe é estatisticamente igual entre os anos analisados, as demais variáveis são estatisticamente diferentes.

Fonte: Elaboração própria com base em dados da pesquisa. 
A região com maior proporção de trabalhadores com segunda ocupação é a região Nordeste, seguida da Sudeste em 2004. Já em 2014, ocorre uma inversão, Sudeste passa a ter um maior percentual.

Em relação à escolaridade, mensurada pelos anos de estudo, os indivíduos analisados possuem nível de escolaridade acima da média nacional, que gira em torno de 7,5 anos de estudo (BRASIL, 2014). O aumento do nível é compreensível, uma vez que o mercado de trabalho vem exigindo maiores níveis de capital humano.

A Tabela 3 expõe as médias das variáveis que caracterizam os trabalhos, ${ }^{7}$ tanto o principal quanto o secundário. Considere que o Rendimento do Trabalho Principal (W1) e o Rendimento do Trabalho Secundário (W2) foram corrigidos pelo IPCA, a preços constantes de setembro de $2014 .^{8}$

Tabela 3 - Características do trabalho, Brasil, 2004/2014

\begin{tabular}{lccccccccc}
\hline & \multicolumn{4}{c}{$\mathbf{2 0 0 4}$} \\
\cline { 2 - 10 } Características & \multicolumn{2}{c}{$\begin{array}{c}\text { Apenas uma } \\
\text { ocupação }\end{array}$} & \multicolumn{2}{c}{ Duas ocupações } & \multicolumn{2}{c}{$\begin{array}{c}\text { Apenas uma } \\
\text { ocupação }\end{array}$} & \multicolumn{2}{c}{ Duas ocupações } \\
\cline { 2 - 10 } & Média & DP & Média & DP & Média & DP & Média & DP \\
\hline H1 & 40,90 & 14,31 & 34,50 & 13,96 & 38,87 & 13,29 & 33,63 & 13,62 \\
W1 & 832,87 & 1529,637 & 1072,36 & 2058,74 & 1695,68 & 2575,48 & 2226,18 & 4650,40 \\
H2 & - & - & 19,87 & 11,80 & - & - & 19,68 & 12,22 \\
W2 & - & - & 656,54 & 1149,33 & - & - & 1433,51 & 2326,26 \\
Privado & 0,79 & 0,41 & 0,47 & 0,50 & 0,80 & 0,40 & 0,50 & 0,50 \\
Formal & 0,58 & 0,49 & 0,51 & 0,50 & 0,69 & 0,46 & 0,61 & 0,49 \\
Estabilidade & 6,95 & 8,57 & 9,60 & 9,27 & 8,11 & 10,37 & 9,59 & 9,83 \\
\hline
\end{tabular}

Nota: DP = desvio-padrão.

Fonte: Elaboração própria com base em dados da pesquisa.

Em relação ao rendimento do trabalho principal, para aqueles que estão alocados no mercado secundário esse rendimento é maior em ambos os anos. Essa característica pode estar associada aos maiores níveis de capital humano dos moonlighters. Contudo, isso também pode indicar a presença de benefícios não pecuniários na escolha de ter uma segunda ocupação, como visto em Böheim e Taylor (2004).

7 O teste de diferença das médias (teste $t$ ) foi realizado para todas as variáveis. Apenas a estabilidade mostrou-se estatisticamente igual entre os anos analisados.

8 Índice de Preços ao Consumidor Amplo (IPCA). Utilizou-se o procedimento de extrapolação adotado por Freguglia (2007). 
Em relação ao setor de trabalho da ocupação principal, se privado ou público, a proporção de indivíduos do setor privado com segunda ocupação é menor do que para aqueles com apenas uma ocupação. De acordo com Casari e Bacha (2011), esse resultado pode sugerir que os trabalhadores busquem um emprego público visando a uma maior estabilidade e mantêm um segundo emprego por questões de satisfação pessoal.

Quanto à estabilidade, esta é maior para aqueles com duas ocupações, fato que não condiz com os aspectos teóricos, no sentido de que uma das causas destacadas para se ter um segundo emprego é diminuir os riscos associados à perda da ocupação principal. Entretanto, se combinarmos essa informação com o fato supracitado, de que os moonlighters têm maior nível de escolaridade, tal resultado pode se justificar. Afinal, seria coerente relacionar maior escolaridade a maior produtividade, e esta, por sua vez, pode implicar maior estabilidade.

Quanto à composição por grupo de atividade econômica dos empregos, a Tabela 4 expõe a distribuição percentual. Verifica-se, pela análise da composição por setor de atividade, que não há diferenças marcantes na distribuição dos setores para aqueles que estão inseridos ou não no mercado de múltiplas ocupações. Destaca-se a maior concentração no setor de serviços, fato que elucida o padrão de configuração do mercado.

Tabela 4 - Setor de atividade econômica, Brasil, 2004/2014 (em \%)

\begin{tabular}{|c|c|c|c|c|c|c|}
\hline \multirow{3}{*}{ Setor } & \multicolumn{3}{|c|}{2004} & \multicolumn{3}{|c|}{2014} \\
\hline & \multirow{2}{*}{$\begin{array}{c}\text { Uma } \\
\text { ocupação }\end{array}$} & \multicolumn{2}{|c|}{ Duas ocupações } & \multirow{2}{*}{$\begin{array}{c}\text { Uma } \\
\text { ocupação }\end{array}$} & \multicolumn{2}{|c|}{ Duas ocupações } \\
\hline & & Principal & Secundária & & Principal & Secundária \\
\hline Agrícola & 19,10 & 24,73 & 25,14 & 14,63 & 12,47 & 15,83 \\
\hline Indústria & 21,88 & 11,42 & 11,24 & 22,65 & 12,49 & 12,07 \\
\hline $\begin{array}{l}\text { Administração } \\
\text { Pública }\end{array}$ & 4,97 & 8,92 & 3,76 & 5,09 & 7,58 & 3,61 \\
\hline Serviços & 46,75 & 48,33 & 47,83 & 48,86 & 59,61 & 59,98 \\
\hline Outras Atividades & 7,30 & 6,60 & 12,03 & 8,77 & 7,85 & 8,51 \\
\hline
\end{tabular}

Fonte: Elaboração própria com base em dados da pesquisa.

Verifica-se, pela análise da composição por setor de atividade, que não há diferenças marcantes na distribuição dos setores para aqueles que estão alocados ou não com ocupações adicionais.

\subsection{ESTIMATIVA DA OFERTA DE TRABALHO}

A Tabela 5 expõe os resultados estimados da função de oferta de trabalho (efeitos marginais) no mercado de trabalho secundário. Os efeitos marginais estimados demons- 
tram as relações entre as variáveis independentes e a oferta. ${ }^{9}$ Em 2004 as variáveis indicativas de sexo e raça não obtiveram significância estatística. Entretanto, para o ano de 2014 observou-se que ser do sexo masculino aumenta a oferta de trabalho e ser de cor branca a reduz.

Ser do sexo masculino em 2014 aumenta a oferta em 0,44 horas semanais, resultado semelhante ao de Foley (1997) e Martinez Jr. et al. (2014). Ainda não há um consenso na literatura sobre o efeito do gênero na oferta e/ou participação no mercado de trabalho secundário, pois os trabalhos de Casari e Bacha (2011) e Menezes e Carrera-Fernandez (2003) indicam uma maior probabilidade para as mulheres.

Tabela 5 - Estimativa da função de oferta de trabalho: efeito marginal, Brasil, 2004/2014

\begin{tabular}{|c|c|c|c|c|}
\hline \multirow{2}{*}{$\begin{array}{l}\text { Variável Dependente: } \\
\text { H2 }\end{array}$} & \multicolumn{2}{|c|}{2004} & \multicolumn{2}{|c|}{2014} \\
\hline & $\mathrm{dy} / \mathrm{dx}$ & Erro-padrão & $\mathrm{dy} / \mathrm{dx}$ & Erro-padrão \\
\hline \multicolumn{5}{|c|}{ Características Individuais } \\
\hline Sexo & $-0,366$ & 0,258 & $0,438^{*}$ & 0,153 \\
\hline Raça & $-0,202$ & 0,141 & $-0,200^{* * *}$ & 0,115 \\
\hline Idade & $0,267^{*}$ & 0,045 & $0,324^{*}$ & 0,029 \\
\hline Idade2 & $-0,003^{*}$ & 0,001 & $-0,004^{*}$ & 0,000 \\
\hline Estudo (1-3) & 0,230 & 0,421 & $-0,335$ & 0,489 \\
\hline Estudo (4-7) & $-0,047$ & 0,354 & 0,431 & 0,391 \\
\hline Estudo (8-10) & 0,057 & 0,378 & $0,726^{\star * *}$ & 0,394 \\
\hline Estudo (11-14) & 0,557 & 0,364 & $1,172^{*}$ & 0,380 \\
\hline Estudo (15 oumais) & $3,635^{*}$ & 0,517 & $3,391^{*}$ & 0,490 \\
\hline \multicolumn{5}{|c|}{ Características da Família } \\
\hline H_chefe & $1,532^{*}$ & 0,249 & $1,307^{*}$ & 0,159 \\
\hline M_chefe & $0,906^{*}$ & 0,232 & $1,256^{*}$ & 0,168 \\
\hline N_cri & $-0,048$ & 0,091 & 0,108 & 0,078 \\
\hline N_des & $-0,173$ & 0,206 & 0,029 & 0,178 \\
\hline \multicolumn{5}{|c|}{ Características da Ocupação Principal } \\
\hline Privado & $-0,860^{*}$ & 0,248 & $-1,186^{*}$ & 0,197 \\
\hline Formal & $-0,128$ & 0,155 & $-0,282^{* *}$ & 0,133 \\
\hline Estabilidade (anos) & $-0,006$ & 0,010 & $0,016^{* *}$ & 0,008 \\
\hline Horas Principal & $-0,096^{*}$ & 0,006 & $-0,091^{*}$ & 0,005 \\
\hline
\end{tabular}

9 Os coeficientes do modelo tobit encontram-se no Apêndice B. 
Tabela 5 - Estimativa da função de oferta de trabalho: efeito marginal, Brasil, 2004/2014

(Cont.)

\begin{tabular}{|c|c|c|c|c|}
\hline \multirow{2}{*}{$\begin{array}{l}\text { Variável Dependente: } \\
\text { H2 } \\
\end{array}$} & \multicolumn{2}{|c|}{2004} & \multicolumn{2}{|c|}{2014} \\
\hline & $d y / d x$ & Erro-padrão & $\mathrm{dy} / \mathrm{dx}$ & Erro-padrão \\
\hline \multicolumn{5}{|c|}{ Setor de Atividade Econômica da Ocupação Principal } \\
\hline Agrícola & $-2,321^{* * *}$ & 1,294 & $-0,860$ & 1,028 \\
\hline Indústria & $-1,170^{*}$ & 0,245 & $-0,908^{*}$ & 0,197 \\
\hline Administração Pública & 0,008 & 0,357 & $-0,459^{* * *}$ & 0,278 \\
\hline Serviços & 0,168 & 0,218 & $0,481^{*}$ & 0,168 \\
\hline \multicolumn{5}{|c|}{ Características de Residência } \\
\hline Migrante & $-0,236^{* * *}$ & 0,140 & $-0,041$ & 0,109 \\
\hline RM & $-1,100^{*}$ & 0,145 & $-1,179^{*}$ & 0,120 \\
\hline Urb & $-0,091$ & 0,288 & $-0,018$ & 0,254 \\
\hline Centro-Oeste & $-0,005$ & 0,221 & $-0,180$ & 0,189 \\
\hline Norte & 0,132 & 0,260 & $-1,400^{*}$ & 0,176 \\
\hline Nordeste & $0,451^{* *}$ & 0,191 & 0,102 & 0,148 \\
\hline Sul & 0,083 & 0,188 & $0,509^{*}$ & 0,164 \\
\hline Renda do Não Trabalho & $-0,0005^{* *}$ & 0,00025 & 0,0001 & 0,00009 \\
\hline
\end{tabular}

Nota: $\left(^{* * *}\right) p<0,10,\left(^{* *}\right) p<0,05,\left({ }^{*}\right) p<0,01$, onde $p=p$-valor.

Fonte: Elaboração própria com base em dados da pesquisa.

Ainda em relação às características individuais, temos que a idade aumenta a oferta. Porém, tal efeito ocorre a taxas decrescentes. Uma variação de um ano de idade representa ampliação da oferta em 0,27 horas/semanais em 2004 e de 0,32 em 2014.

No que compete às dummies de educação, em 2004 observou-se um aumento de oferta somente a partir de 15 anos de estudo. Em 2014 consegue-se avaliar um aumento dessa importância a partir do grupo de 8 a 10 anos de estudo, indicando que a oferta aumenta com o aumento da escolaridade em relação à categoria de referência. Esse comportamento da educação é verificado em diversas pesquisas internacionais, como Foley (1997) e Panos, Pouliakas e Zangelidis (2014). Do efeito marginal, para o grupo com 15 anos ou mais de estudo, há um aumento na oferta semanal de 3,6 horas em 2004 e de 3,4 horas em 2014.

Dentre as variáveis que caracterizam o grupo familiar, ambas as variáveis para chefe de família relacionam-se de forma positiva com a oferta. Esse é um resultado esperado, pois ser chefe de família traz consigo a responsabilidade pela manutenção do lar, seja por meio de custos habitacionais e/ou de educação. Para homens chefes de família a oferta caiu de 2004 para 2014, já para as mulheres a oferta aumentou, passando de 0,9 para 1,2 horas. 
O resultado para mulheres apontado no parágrafo anterior pode estar relacionado com o aumento de mulheres no comando dos lares no Brasil. Em 2000 as mulheres representavam 22,2\% dos chefes de família e, em 2010, esse percentual era de 37,3\%, de acordo com dados do Censo Demográfico (IBGE, 2010).

Pessoas alocadas em trabalhos principais no setor privado tendem a ofertar menos horas de trabalho no mercado secundário. Isso pode estar relacionado com o número de horas trabalhadas por semana ou a estrutura dos contratos firmados que podem impossibilitar a alocação de mais horas em outra ocupação. Resultado semelhante foi encontrado por Wu, Braimbridge e $\mathrm{Zu}$ (2009).

A relação negativa do setor formal na oferta de trabalho pode ser atribuída à diminuição do grau de informalidade que aconteceu nos últimos, o que pode tornar os agentes menos propensos à busca de outras fontes de renda. Por exemplo, a informalidade passou de 57,4\% em 2003 para 47\% em 2012, de acordo com Vargas (2015).

Duas variáveis foram inseridas no modelo para tentar captar dois dos motivos teóricos expostos na subseção 2.1: restrição de horas e insegurança na ocupação principal, as variáveis utilizadas foram o número de horas trabalhadas semanalmente no emprego principal e o número de anos de serviço, como proxy para a insegurança.

O motivo restrição de horas foi captado nos dois anos em análise, indicando que quanto mais tempo a pessoa passa na ocupação principal, menos horas ela dispõe para alocar no mercado secundário. Apesar de ser baixo, o efeito marginal da restrição de horas no trabalho principal corrobora com a teoria.

A variável que capta o efeito insegurança no mercado de trabalho nos diz que o aumento de um ano na estabilidade do agente eleva em 0,016 a oferta de horas em 2014, apesar de o efeito ser baixo, mostra que estar alocado no mercado secundário relaciona-se de forma positiva com a estabilidade adquirida em seu emprego principal. Essa evidência é contrária aos resultados de Bell, Hart e Wright (1997).

Apenas o setor de serviços relaciona-se de forma positiva com a oferta de trabalho secundário. Em 2014, estar nesse setor aumenta a oferta num valor aproximado de 0,5 horas semanais, em comparação com outras atividades (categoria omitida). Esse resultado pode estar relacionado com a composição desse grupo, ${ }^{10}$ em que estão incluídos: ambulantes de alimentação, cabeleireiros, atividades de rádio e televisão que podem tornar horários mais flexíveis e influenciando de forma positiva a oferta de trabalho numa segunda ocupação. De toda forma, seria necessária uma análise mais detalhada da composição dos demais agrupamentos de atividades para outras conclusões. Além do mais, é o setor com maior representatividade entre as categorias de atividades, como observado na Tabela 4.

${ }^{10}$ O IBGE fornece o detalhamento dos agrupamentos de atividade juntamente com os microdados da PNAD. 
Pela análise do perfil demográfico do moonlighter, os migrantes representam $36 \%$ da amostra em 2004 e 53\% em 2014, logo, espera-se que essa característica impacte de forma positiva na oferta de trabalho. Entretanto, em 2004 houve redução da oferta devido a essa característica. Para apontamentos mais acurados em relação a esse aspecto há a necessidade de uma maior reflexão sobre a composição das ocupações dos migrantes e dos locais onde estão inseridos. Contudo, isso foge do escopo desta pesquisa.

Da análise das regiões de residência, nota-se um aumento de oferta, em 2004, para os nordestinos em relação aos residentes do Sudeste (categoria-base). Em 2014, nota-se uma relação positiva dos residentes da região Sul e negativa dos residentes da região Norte. Tal resultado pode ser explicado pela dinamicidade e/ou pelo nível médio de escolaridade exigido nessas economias. Atenta-se ao fato de que mais fatores podem afetar esses resultados. Contudo, não se consegue captá-los apenas nas dummies de região. ${ }^{11}$

O sinal negativo do rendimento do não trabalho em 2004 revela a importância econômica do lazer, pois, quanto maior essa renda, menor a oferta no mercado secundário, como destacado na seção 2.3, no modelo teórico. É importante destacar que para atender a alguns aspectos teóricos, restrições foram impostas à amostra. Em pesquisas posteriores pode-se adequar o modelo teórico à inclusão de todos os tipos de trabalhadores, assim como realizado por Atherton et al. (2016), que inclui a possibilidade de o trabalhador atuar por conta própria nos dois empregos, por exemplo.

Por fim, a Tabela 7, no Apêndice C, revela a importância da adoção do plano amostral complexo no procedimento de estimação do modelo tobit. Na maioria dos casos, tanto o valor do DEFF como do MEFF têm valores maiores que a unidade, indicando, dessa forma, que a desconsideração do plano amostral implica em subestimação da variância.

\section{CONSIDERAÇÕES FINAIS}

O presente estudo analisou os determinantes da oferta de trabalho no mercado de trabalho secundário no Brasil nos anos de 2004 e 2014. A principal análise baseou-se em um modelo de oferta de trabalho e de um conjunto de variáveis socioeconômicas que incidem sobre a decisão de oferta de trabalho do agente.

$\mathrm{Na}$ análise descritiva verificou-se que os indivíduos pertencentes ao mercado do múltiplo emprego são compostos em sua maioria por homens, chefes de família e tem

11 Uma possibilidade para entender melhor o mercado de múltiplas ocupações no Brasil é desagregar as regiões e estudar a oferta e/ou participação para cada uma delas. 
um nível de educação mais elevado do que aqueles que possuem apenas uma ocupação. Não se observou nenhum padrão em relação à distribuição geográfica.

Há vantagem salarial, da ocupação principal, para trabalhadores alocados no mercado secundário. Tal resultado pode estar associado aos maiores níveis educacionais, que impactam os rendimentos de ambas ocupações.

Em contraste aos resultados de Menezes e Carrera-Fernandez (2003) e Casari e Bacha (2011), constatou-se que ser do gênero masculino aumenta a oferta de trabalho na segunda ocupação. A idade aumenta a oferta, contudo as taxas decrescem. Da análise dos grupos educacionais, percebe-se um aumento de oferta para os níveis mais elevados. Além disso, ao contrário de Casari e Bacha (2011), utilizam-se agrupamentos de atividades como controles adicionais para oferta de trabalho.

Estar no setor privado reduz a oferta, assim como ter ocupação principal formal. Outros fatores que impactam de forma negativa são ser migrante e residir em região metropolitana. A inserção das variáveis de setor de atividade econômica leva a concluir que ter ocupação principal definida no setor de serviços aumenta a oferta de trabalho.

Como aspectos teóricos não foram testados por Menezes e Carrera-Fernandez (2003), tomou-se o cuidado de inserir variáveis que justificassem tanto a restrição de horas como o motivo insegurança na ocupação principal. O motivo insegurança na ocupação principal captado pela variável estabilidade não obteve o sinal esperado e comportou-se de forma a aumentar a oferta de trabalho. Esse resultado, contudo, é semelhante ao de Casari e Bacha (2011). Já a restrição de horas comportou-se como na literatura internacional, e é um fator que reduz a oferta de trabalho.

Em suma, os resultados encontrados sugerem a relevância de fatores socioeconômicos como determinantes da oferta de trabalho no mercado do múltiplo emprego, além de reforçar que esse é um fenômeno presente no mercado de trabalho brasileiro. Os policymakers devem estar cientes da existência desse contingente populacional a fim de considerarem as peculiaridades do mercado de trabalho no Brasil na concepção de políticas, específicas ou não. Ademais, estudos futuros podem analisar os moonlighters sob perspectivas complementares, como análises dos quantis de rendimentos ou grupos específicos como o dos migrantes, por exemplo.

\section{REFERÊNCIAS}

ATHERTON, A.; FARIA, J. R.; HEATLEY, D.; WU, D.; WU, Z. The decision to moonlight: does second job holding by the self-employed and employed differ? Industrial Relations Journal, v. 47, n. 3, p. 279-299, 2016.

AVERETT, S. L. Moonlighting: multiple motives and gender differences. Applied Economics, v. 33, n. 11, p. 1391-1410, 2001. 
BELL, D.; HART, R.; WRIGHT, R. Multiple job holding as a "hedge" against unemployment. CEPR Discussion Papers, n. 1626, 1997.

BÖHEIM, R.; TAYLOR, M. P. And in the evening she's a singer with the band - second jobs, plight or pleasure? Discussion Papers Series, Institute for the Study of Labor (IZA), n. 1081, 2004. Disponível em: <ftp://repec.iza.org/RePEc/Discussionpaper/dp1081.pdf> Acesso em: 20 mai 2016.

BRASIL. Ministério da Educação. Pesquisa aponta queda do analfabetismo e aumento na escolaridade. Brasília, 2014. Disponível em: <http://portal.mec.gov.br/component/tags/ tag/32962>. Acesso em: 15 jan. 2017.

CARVALHO, A. P.; NÉRI, M.; SILVA, D. B. N. Diferenciais de salários por raça e gênero no Brasil: aplicação dos procedimentos de Oaxaca e Heckman em pesquisas amostrais complexas. Rio de Janeiro: IBGE, 2006. Mimeo.

CASARI, P.; BACHA, C. J. C. Oferta de trabalho no Brasil: uma análise do segundo emprego. In: ENCONTRO NACIONAL DE ECONOMIA ANPEC, 39., Foz do Iguaçu, PR, 6-9 dez. 2011. Anais.... Foz do Iguaçu: ANPEC, 2011.

CONWAY, K. S.; KIMMEL, J. Male labor supply estimates and the decision to moonlight. Labour Economics, v. 5, n. 2, p. 135-166, 1998.

FOLEY, M. C. Multiple job holding in Russia during economic transition. Center Discussion Paper, Economic Growth Center, Yale University, n. 781, 1997.

FREGUGLIA, R. S. Efeitos da migração sobre os salários no Brasil. 2007. Tese (Doutorado em Teoria Econômica) - Faculdade de Economia, Administração e Contabilidade, Universidade de São Paulo, São Paulo, SP, Brasil, 2007.

GUARIGLIA, A.; KIM, B. The dynamics of moonlighting in Russia. Economics of Transition, v. 14, n. 1, p. 1-45, 2006.

HEINECK, G. The determinants of secondary jobholding in Germany and the UK. Zeitschriftfür Arbeitsmarkt Forschung, v. 42, n. 2, p. 107-120, 2009.

HEINECK, G.; SCHWARZE, J. Fly me to the moon: the determinants of secondary jobholding in Germany and the UK. Discussion Papers Series, Institute for the Study of Labor (IZA), n. 1358, 2004. Disponível em: <ftp.iza.org/dp1358.pdf>. Acesso em: 10 ago. 2016.

IBGE - INSTITUTO BRASILEIRO DE GEOGRAFIA E ESTATÍSTICA. Censo Demográfico 2010 - Famílias e Domicílios: Resultados da Amostra. Rio de Janeiro: IBGE, 2010. Disponível em: <http://biblioteca.ibge.gov.br/visualizacao/periodicos/97/cd_2010_familias_domicilios_amostra.pdf>. Acesso em: 20 nov. 2016.

KIMMEL, J.; CONWAY, K.S. Who moonlights and why? Evidence from the SIPP. Industrial Relations: A Journal of Economy and Society, v. 40, n. 1, p. 89-120, 2001.

LEONE, E. T.; MAIA, A. G.; BALTAR, P. E. Mudanças na composição das famílias e impactos sobre a redução da pobreza no Brasil. Economia e Sociedade, v. 19, n. 1, p. 59-77, 2010.

MARTINEZ JR., A. et al. Multiple job holding and income mobility in Indonesia. Research in Social Stratification and Mobility, v. 37, p. 91-104, 2014. 
MENEZES, W. F.; CARRERA-FERNANDEZ, J. Necessidades e os condicionantes da segunda ocupação. Análise Econômica, v. 21, n. 39, p. 189-209, 2003.

PANOS, G. A.; POULIAKAS, K.; ZANGELIDIS, A. Multiple job holding, skill diversification, and mobility. Industrial Relations: A Journal of Economy and Society, v. 53, n. 2, p. 223-272, 2014.

RENNA, F. Moonlighting and overtime: a cross-country analysis. Journal of Labor Research, v. 27, n. 4, p. 575-591, 2006.

SANTOS, G. C. Inserção e rendimentos no mercado de trabalho brasileiro. Dissertação (Mestrado em Economia) - Programa de Pós-Graduação em Economia, Universidade Federal de Viçosa, Viçosa, MG, Brasil, 2009.

SHISHKO, R.; ROSTKER, B. The economics of multiple job holding. The American Economic Review, p. 298-308, 1976.

SILVA, P. L. N.; PESSOA, D. G. C.; LILA, M. F. Análise estatística de dados da PNAD: incorporando a estrutura do plano amostral. Ciência \& Saúde Coletiva, v. 7, n. 4, p. 659-670, 2002.

VARGAS, J. A informalidade no mercado de trabalho: um desafio institucional permanente para a economia brasileira. Dissertação (Mestrado em Economia) - Programa de Pós-Graduação em Economia, Universidade Federal do Espírito Santo, Vitória, ES, Brasil, 2015.

WU, Z.; ZHU, Y.; BAIMBRIDGE, M. Multiple job holding in the United Kingdom: Evidence from the British household panel survey, Applied Economics, v. 41, n. 21, p. 2751-2766, 2009. 


\section{APÊNDICE A - DESCRIÇÃO DAS VARIÁVEIS}

\section{Quadro 1 - Descrição das Variáveis}

\begin{tabular}{|c|c|}
\hline Variável & Descrição \\
\hline \multicolumn{2}{|l|}{ Dependente } \\
\hline $\mathrm{H} 2$ & $\begin{array}{l}\text { Número de horas trabalhadas semanalmente na segunda ocupação; sendo igual a zero } \\
\text { para os indivíduos que possuem apenas uma ocupação e um valor positivo }(\mathrm{H} 2>0) \text { para } \\
\text { aqueles alocados no mercado secundário }\end{array}$ \\
\hline \multicolumn{2}{|l|}{ Independentes } \\
\hline Sexo & $=1$ se do sexo masculino, 0 se do sexo feminino \\
\hline Raça & $=1$ se da cor branca, 0 se das demais raças \\
\hline Idade & Idade do indivíduo, medida em anos \\
\hline Idade2 & Termo quadrático da idade \\
\hline Estudo & $\begin{array}{l}\text { Dummies para os seguintes grupos de anos de estudo: } \\
\text { Sem instrução e menos de } 1 \text { ano (grupo base); 1-3 anos de estudo; } 4-7 \text { anos de estudo; } \\
8-10 \text { anos de estudo; } 11-14 \text { anos de estudo; } 15 \text { anos de estudo ou mais. }\end{array}$ \\
\hline H_chefe & $=1$ se chefe da família do sexo masculino, 0 caso contrário \\
\hline M_chefe & $=1$ se chefe da família do sexo feminino, 0 caso contrário \\
\hline N_cri & Número de crianças na família com idade igual ou menor a 5 anos \\
\hline N_des & Número de desempregados na família \\
\hline Privado & $=1$ se o setor do emprego principal é privado, 0 caso contrário \\
\hline Formal & $=1$ se tem carteira assinada no emprego principal, 0 caso contrário \\
\hline $\begin{array}{l}\text { Setor de atividade da } \\
\text { ocupação principal }\end{array}$ & $\begin{array}{l}\text { Para indicar o setor de atividade foram criadas dummies para os setores de Agricultura, } \\
\text { Indústria, Administração Pública, Serviços e Outras Atividades (grupo base) }\end{array}$ \\
\hline Horas Principal (H1) & Número de horas trabalhadas semanalmente no trabalho principal \\
\hline Estabilidade & Número de anos na ocupação principal \\
\hline Migrante & = 1 se migrante (em relação a UF de nascimento), 0 caso contrário \\
\hline $\mathrm{RM}$ & $=1$ se reside em região metropolitana, 0 caso contrário \\
\hline Urbano & $=1$ se reside em zona urbana, 0 caso contrário \\
\hline Região de residência & Dummies para as regiões Norte, Nordeste, Sul, Centro-Oeste e Sudeste (grupo base) \\
\hline $\begin{array}{l}\text { Rendimento do Não } \\
\text { trabalho }\end{array}$ & Rendimento de todas as fontes subtraído do rendimento do trabalho \\
\hline
\end{tabular}

Fonte: Elaboração própria com base em dados da pesquisa. 


\section{APÊNDICE B - MODELO TOBIT ESTIMADO}

Tabela 6 - Oferta de trabalho - Brasil - 2004/2014

Variável Dependente: Horas trabalhadas semanalmente na segunda ocupação (H2)

\begin{tabular}{|c|c|c|c|c|}
\hline & \multicolumn{2}{|c|}{2004} & \multicolumn{2}{|c|}{2014} \\
\hline & Coeficiente & Erro-padrão & Coeficiente & Erro-padrão \\
\hline \multicolumn{5}{|c|}{ Características Individuais } \\
\hline Sexo & $-3,213$ & 2,249 & $4,081^{*}$ & 1,430 \\
\hline Raça & $-1,783$ & 1,243 & $-1,853^{* * *}$ & 1,062 \\
\hline Idade & $2,358^{*}$ & 0,400 & $2,999^{*}$ & 0,271 \\
\hline Idade2 & $-0,0278^{*}$ & 0,00517 & $-0,0365^{*}$ & 0,00354 \\
\hline Estudo (1-3) & 2,006 & 3,630 & $-3,161$ & 4,687 \\
\hline Estudo (4-7) & $-0,419$ & 3,134 & 3,925 & 3,506 \\
\hline Estudo (8-10) & 0,503 & 3,322 & $6,568^{* * *}$ & 3,475 \\
\hline Estudo (11-14) & 4,863 & 3,144 & $10,80^{*}$ & 3,482 \\
\hline Estudo (15 ou mais) & $27,34^{*}$ & 3,330 & $27,69^{*}$ & 3,530 \\
\hline \multicolumn{5}{|c|}{ Características da Família } \\
\hline H_chefe & $13,43^{*}$ & 2,174 & $11,79^{*}$ & 1,398 \\
\hline M_chefe & $7,680^{*}$ & 1,883 & $11,07^{*}$ & 1,415 \\
\hline N_cri & $-0,421$ & 0,800 & 0,997 & 0,723 \\
\hline N_des & $-1,524$ & 1,818 & 0,270 & 1,645 \\
\hline \multicolumn{5}{|c|}{ Características da Ocupação Principal } \\
\hline Privado & $-7,298^{*}$ & 2,013 & $-10,45^{*}$ & 1,656 \\
\hline Formal & $-1,127$ & 1,362 & $-2,597^{* *}$ & 1,210 \\
\hline Estabilidade (anos) & $-0,0507$ & 0,0924 & $0,147^{\star *}$ & 0,0745 \\
\hline Horas Principal & $-0,851^{*}$ & 0,0587 & $-0,846^{*}$ & 0,0507 \\
\hline \multicolumn{5}{|c|}{ Setor de Atividade Econômica da Ocupação Principal } \\
\hline Agrícola & $-23,70$ & 15,34 & $-8,356$ & 10,47 \\
\hline Indústria & $-10,52^{*}$ & 2,252 & $-8,575^{*}$ & 1,901 \\
\hline Adm. Pública & 0,0739 & 3,146 & $-4,354$ & 2,696 \\
\hline Serviços & 1,486 & 1,929 & $4,459^{*}$ & 1,567 \\
\hline
\end{tabular}


Tabela 6 - Oferta de trabalho - Brasil - 2004/2014

(Cont.)

Variável Dependente: Horas trabalhadas semanalmente na segunda ocupação (H2)

\begin{tabular}{|c|c|c|c|c|}
\hline & \multicolumn{2}{|c|}{2004} & \multicolumn{2}{|c|}{2014} \\
\hline & Coeficiente & Erro-padrão & Coeficiente & Erro-padrão \\
\hline \multicolumn{5}{|c|}{ Características de Residência } \\
\hline Migrante & $-2,086^{\star * *}$ & 1,247 & $-0,383$ & 1,005 \\
\hline $\mathrm{RM}$ & $-9,848^{*}$ & 1,305 & $-11,09^{*}$ & 1,145 \\
\hline Urb & $-0,796$ & 2,517 & $-0,164$ & 2,351 \\
\hline Centro-Oeste & $-0,0434$ & 1,951 & $-1,677$ & 1,778 \\
\hline Norte & 1,154 & 2,264 & $-13,90^{*}$ & 1,851 \\
\hline Nordeste & $3,913^{* *}$ & 1,639 & 0,940 & 1,361 \\
\hline Sul & 0,727 & 1,648 & $4,635^{*}$ & 1,469 \\
\hline R. do Não Trabalho & $-0,00438^{* *}$ & 0,00222 & 0,00107 & 0,000865 \\
\hline Constante & $-79,42^{*}$ & 8,813 & $-114,4^{*}$ & 6,536 \\
\hline Sigma & $39,56^{*}$ & 0,825 & $43,94^{*}$ & 0,776 \\
\hline Estatística F & \multicolumn{2}{|c|}{42.45} & \multicolumn{2}{|c|}{50.68} \\
\hline Prob $>$ F & \multicolumn{2}{|c|}{0.000} & \multicolumn{2}{|c|}{0.000} \\
\hline Obs. sem censura & 1245 & & 2115 & \\
\hline$N$ & 37198 & & 85493 & \\
\hline População & 17.062 .592 & & 48.118 .874 & \\
\hline
\end{tabular}

Nota: (a) $\left(^{* * *}\right) p<0.10,\left({ }^{* *}\right) p<0.05,\left({ }^{*}\right) p<0.01$, onde $p=p$-valor. (b) Extrapolando o nível de significância para $12 \%$ as variáveis Agrícola e Estudo (11-14) em 2004 e Administração Pública em 2014 são estatisticamente significantes.

Fonte: Elaboração própria com base em dados da pesquisa. 
APÊNDICE C - EFEITO DO PLANO AMOSTRAL NO MODELO TOBIT

Tabela 7- Efeito do plano amostral - Brasil - 2004/2014

\begin{tabular}{|c|c|c|c|c|}
\hline \multirow{2}{*}{ Variáveis } & \multicolumn{2}{|c|}{2004} & \multicolumn{2}{|c|}{2014} \\
\hline & DEFF & MEFF & DEFF & MEFF \\
\hline \multicolumn{5}{|c|}{ Características Individuais } \\
\hline Sexo & 1,181 & 1,152 & 1,194 & 1,121 \\
\hline Raça & 1,091 & 1,084 & 1,348 & 1,238 \\
\hline Idade & 1,355 & 1,283 & 1,108 & 1,119 \\
\hline Idade2 & 1,349 & 1,301 & 1,104 & 1,170 \\
\hline Estudo (1-3) & 1,226 & 1,246 & 1,138 & 1,206 \\
\hline Estudo (4-7) & 1,171 & 1,222 & 1,008 & 1,173 \\
\hline Estudo (8-10) & 1,215 & 1,275 & 0,997 & 1,169 \\
\hline Estudo (11-14) & 1,201 & 1,250 & 1,063 & 1,266 \\
\hline Estudo (15 ou mais) & 1,264 & 1,217 & 1,065 & 1,206 \\
\hline \multicolumn{5}{|l|}{ Características da Família } \\
\hline H_chefe & 1,161 & 1,120 & 1,151 & 1,166 \\
\hline M_chefe & 1,108 & 1,093 & 1,113 & 1,040 \\
\hline N_cri & 1,345 & 1,287 & 1,311 & 1,284 \\
\hline N_des & 1,266 & 1,572 & 1,333 & 1,312 \\
\hline \multicolumn{5}{|c|}{ Características da Ocupação Principal } \\
\hline Privado & 1,297 & 1,306 & 1,457 & 1,307 \\
\hline Formal & 1,203 & 1,217 & 1,267 & 1,274 \\
\hline Horas Principal & 1,243 & 1,158 & 1,337 & 1,320 \\
\hline Estabilidade (anos) & 1,195 & 1,031 & 1,220 & 1,071 \\
\hline \multicolumn{5}{|c|}{ Setor de Atividade Econômica da Ocupação Principal } \\
\hline Agrícola & 1,285 & 1,175 & 0,702 & 0,997 \\
\hline Indústria & 1,269 & 1,412 & 1,374 & 1,444 \\
\hline Administração Pública & 1,306 & 1,271 & 1,222 & 1,207 \\
\hline Serviços & 1,266 & 1,416 & 1,366 & 1,417 \\
\hline \multicolumn{5}{|c|}{ Características de Residência } \\
\hline Migrante & 1,190 & 1,128 & 1,321 & 1,262 \\
\hline RM & 1,177 & 1,240 & 1,390 & 1,457 \\
\hline Urb & 1,057 & 0,971 & 1,558 & 1,433 \\
\hline Centro- Oeste & 0,981 & 1,193 & 1,140 & 1,374 \\
\hline Norte & 1,019 & 1,438 & 0,697 & 1,116 \\
\hline Nordeste & 1,232 & 1,146 & 1,376 & 1,364 \\
\hline Sul & 1,194 & 1,090 & 1,601 & 1,391 \\
\hline Renda do Não Trabalho & 1,114 & 1,723 & 1,076 & 1,389 \\
\hline
\end{tabular}

Fonte: Elaboração própria com base em dados da pesquisa. 